\title{
Controle Descentralizado por Desacoplamento Aplicado a um Módulo Didático Multivariável ${ }^{\star}$
}

\author{
Egydio Tadeu G. Ramos* Anna Paula V. de A. Aguiar* \\ George Acioli Júnior ** Péricles Rezende Barros ${ }^{* *}$ \\ * Departamento de Engenharia Elétrica, Universidade Federal de \\ Campina Grande, $P B$ \\ (e-mails: [egydio.ramos, anna.aguiar]@ee.ufcg.edu.br). \\ ** Departamento de Engenharia Elétrica, Universidade Federal de \\ Campina Grande, $P B$ \\ (e-mails: [georgeacioli, prbarros]@dee.ufcg.edu.br).
}

\begin{abstract}
In this work, an experimental methodology to design decentralized PI control with decoupling is presented. This methodology is applied to a multivariable didactic temperature module for teaching control theory. The aim is to study interactions in multivariable systems and the design of decentralized PI controllers with inverted decouplers. As advanced topic, a methodology for inverted decoupler evaluation and redesign is discussed. The feasibility of the methodology was validated through experiment performed in the module.

Resumo: Neste trabalho, é apresentada uma metodologia experimental para o projeto de controle PI descentralizado por desacoplamento. Esta metodologia é aplicada a um módulo didático de temperatura para ensino da teoria de controle. O objetivo é estudar as interações em sistemas multivariáveis e o projeto de controladores PI descentralizados com desacoplador invertido. Como tópico avançado, uma metodologia para avaliação e reprojeto de desacoplador invertido é discutida. A viabilidade da metodologia foi validada por meio de experimentos realizados no módulo.
\end{abstract}

Keywords: Multivariable Systems; Decentralized Control; Decoupler.

Palavras-chaves: Sistemas Multivariáveis; Controle Descentralizado; Desacoplador.

\section{INTRODUÇÃO}

Em muitos processos encontrados na indústria, existe a necessidade de se controlar um conjunto de variáveis de saída obtidos por medições de sensores a partir da manipulação de diversas entradas por meio de atuadores. Esses são sistemas com múltiplas entradas e múltiplas saídas (MIMO, do inglês multiple-input and multiple-output). Uma característica presente nesses processos são as interações ou acoplamentos existentes entre as entradas e as saídas, o que aumenta a complexidade do projeto do sistema de controle. Dessa forma, o engenheiro de controle deve estar apto a trabalhar com sistemas dessa natureza.

Métodos de controle centralizado, como o controle por modelo preditivo (MPC, do inglês model predictive control), possuem desempenho satisfatório, mas seu uso é limitado pela complexidade computacional (Dong et al., 2015).

$\mathrm{Na}$ indústria é comumente utilizado o controle descentralizado, caracterizado por uma matriz diagonal de controladores com estrutura fixa, cada um atuando em uma malha específica do processo. Esta traz como vantagem a simplicidade na implementação pois baseia-se na sintonia

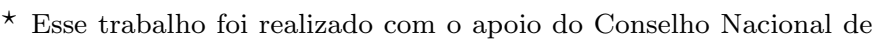
Desenvolvimento Científico e Tecnológico (CNPq).
}

de controladores como o PI, PID, entre outros. Contudo, com essa estrutura nem sempre é possível alcançar o desempenho desejado devido às interações entre as malhas.

Uma alternativa é utilizar o controle por desacoplamento, que consiste na adição de um bloco desacoplador entre o processo e o controlador. Com isso é possível obter o desempenho desejado do controle descentralizado, por meio da redução das interações entre as malhas. Dessa forma, o processo MIMO é visto como um conjunto de malhas de uma entrada e uma saída (SISO, do inglês single-input and single-output), possibilitando a sintonia de controladores independentes para cada malha (Acioli Júnior and Barros, 2011).

Diante da relevância que o controle de sistemas multivariáveis possui em aplicações industriais, este trabalho objetiva apresentar uma metodologia para o projeto de controle PI descentralizado por desacoplamento. Esta é aplicada a um módulo didático de temperatura, apresentado em Lima et al. (2018), possibilitando a execução por alunos de graduação como complemento ao aprendizado de teoria de controle multivariável. Além disso, são apresentadas aplicações de técnicas mais avançadas de controle por desacoplamento, tais como: a avaliação e o reprojeto do desacoplador. 
Este trabalho está organizado da seguinte forma. $\mathrm{Na}$ seção 2, é apresentada uma visão geral acerca do módulo didático de temperatura. Nas seções 3 e 4, são descritas as metodologias adotadas para o controle por desacoplamento e a avaliação e reprojeto do desacoplador, respectivamente. $\mathrm{Na}$ seção 5, os resultados dos experimentos realizados no módulo são apresentados e discutidos. Por fim, as conclusões do trabalho encontram-se na seção 6 .

\section{MÓDULO DIDÁTICO DE TEMPERATURA}

Conforme apresentado em Lima et al. (2018), o módulo didático de temperatura é utilizado para ensino da teoria de controle na disciplina Laboratório de Controle Digital do curso de Engenharia Elétrica da Universidade Federal de Campina Grande.

O módulo consiste em um circuito formado por dois transistores de efeito de campo e dois sensores de temperatura. Quando excitados por sinais modulados em largura de pulso (PWM, do inglês pulse width modulation) os transistores liberam energia na forma de calor, elevando a temperatura dos arredores, que é medida por meio dos sensores. Na Fig. 1, é apresentado um esquema da disposição desses elementos.

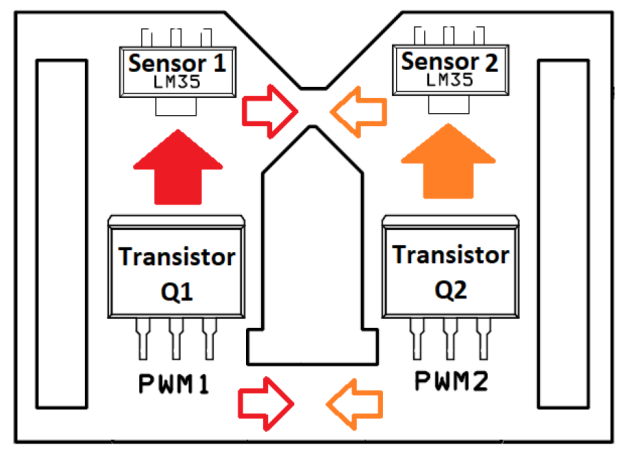

Figura 1. Esquema de disposição dos componentes do módulo de temperatura (Lima et al., 2018).

Esse processo é caracterizado como um sistema de duas entradas e duas saídas (TITO, do inglês two-input and twooutput), cujas entradas são atuadores PWM e as saídas são as temperaturas medidas utilizando os sensores.

Variando o atuador entre 0 e $100 \%$ é possível medir valores de temperaturas numa faixa que compreende desde a temperatura ambiente até cerca de $70^{\circ} \mathrm{C}$.

Devido à distância reduzida entre os componentes, a variação em apenas um atuador modifica a leitura dos dois sensores. Logo, o acoplamento está presente nesse sistema, sendo necessária a implementação do controle por desacoplamento.

\section{METODOLOGIA EXPERIMENTAL}

Nesta seção, é apresentada a metodologia para o projeto do sistema de controle PI com desacoplamento considerando um processo TITO. Sua execução é dividida em quatro etapas:

\section{Etapa 1: Identificação do Processo}

Esta etapa consiste na identificação do processo para a obtenção de um modelo que represente a dinâmica do sistema.

\section{Etapa 2: Emparelhamento de Entradas e Saídas}

Nesta etapa, utiliza-se o modelo obtido para determinar a matriz de ganho relativo (RGA, do inglês relative gain array) do processo, que auxilia na escolha do melhor emparelhamento entrada-saída durante a seleção dos subsistemas SISO.

\section{Etapa 3: Projeto do Desacoplador Invertido}

Nesta etapa, determinam-se as funções de transferência do desacoplador invertido com base no modelo identificado. Esse desacoplador é avaliado e reprojetado de maneira a alcançar um desacoplamento mais efetivo.

\section{Etapa 4: Projeto de Controle PI Descentralizado}

Seguindo o método de controle descentralizado, nesta etapa, são sintonizados controladores PI para as malhas SISO obtidas com o emparelhamento.

\subsection{Identificação do Processo}

Conforme apresentado em (Lima et al., 2018), esta etapa consiste na identificação de um modelo de primeira ordem com atraso (FOPTD, do inglês first order plus time delay), que foi adotado devido às características da resposta do sistema considerado. Assim, sinais do tipo degrau são aplicados a cada entrada de forma sequencial. Em seguida, o método dos mínimos quadrados é aplicado para o cálculo dos parâmetros do modelo representado por:

$$
G(s)=\left[\begin{array}{ll}
\frac{K_{11}}{T_{11} s+1} e^{-L_{11} s} & \frac{K_{12}}{T_{12} s+1} e^{-L_{12} s} \\
\frac{K_{21}}{T_{21} s+1} e^{-L_{21} s} & \frac{K_{22}}{T_{22} s+1} e^{-L_{22} s}
\end{array}\right],
$$

onde $K_{i j}, T_{i j}$ e $L_{i j}$ representam, respectivamente, o ganho, a constante de tempo e o atraso temporal da $i$-ésima saída em resposta a variações na $j$-ésima entrada.

\subsection{Emparelhamento de Entradas e Saídas}

A RGA é um método utilizado para mensurar o acoplamento entre as entradas e saídas em processos MIMO que não possuam ganhos significativos em altas frequências.

O ganho relativo de uma malha é a razão entre o ganho desta quando todas as malhas do sistema estão abertas e o ganho resultante quando apenas a malha considerada está aberta (Bristol, 1966).

A RGA do processo $G, \Lambda(G)$, pode ser calculada por:

$$
\Lambda(G)=G(0) \otimes G^{-T}(0)
$$

onde $G^{-T}$ é a matriz transposta da inversa de $G$ e $\otimes$ representa o produto elemento por elemento das matrizes.

Avaliar cada ganho relativo $\lambda_{i j}$ referente a $i$-ésima saída e a $j$-ésima entrada permite escolher o melhor emparelhamento entrada-saída. Quanto mais próximo de 1 é esse 
valor, menor a interação que essa malha sofre causada pelas demais. Valores negativos indicam que a malha fechada pode levar a instabilidade, e o emparelhamento correspondente deve ser descartado.

\subsection{Projeto do Desacoplador Invertido}

Na literatura são encontradas várias metodologias de projeto para o desacoplador. As estruturas de desacoplamento simplificado (Luyben, 1970) e invertido (Shinskey, 1996) são projetadas a partir de modelos FOPTD do processo. Já no caso de sistemas representados por modelos em espaço de estados, existem outras técnicas para o projeto de desacopladores, como a proposta por Wang (2002).

Neste trabalho, será adotado o esquema com desacoplamento invertido, cuja estrutura é apresentada na Fig. 2, onde $G_{i j}$ é o processo, $D_{i j}$ é o desacoplador e $C_{i}$ é o controlador.

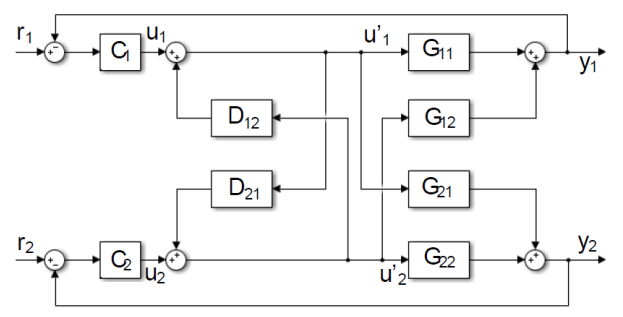

Figura 2. Estrutura de controle descentralizado com inserção do desacoplador invertido (Aguiar, 2018).

O desacoplador deve ser projetado de maneira que o processo desacoplado $(F(s))$ seja uma matriz diagonal dominante, ou seja:

$$
F(s)=G(s) D(s)=\left[\begin{array}{cc}
F_{11}(s) & 0 \\
0 & F_{22}(s)
\end{array}\right] .
$$

Assim, o desacoplador é dado por:

$$
\begin{aligned}
& D_{12}(s)=-\frac{G_{12}(s)}{G_{11}(s)}, \\
& D_{21}(s)=-\frac{G_{21}(s)}{G_{22}(s)} .
\end{aligned}
$$

Considerando a dinâmica do processo descrita em (1), o desacoplador é projetado substituindo (1) em (4) e (5). Assim:

$$
D_{i j}(s)=-K_{i}\left(\frac{T_{i i} s+1}{T_{i j} s+1}\right) e^{-\theta_{i} s},
$$

onde $i, j=1,2, \quad i \neq j$, o ganho $K_{i}=K_{i j} / K_{i i}$, o atraso temporal é $\theta_{i}=\max \left(0, L_{i j}-L_{i i}\right)$ de maneira a garantir que o sistema seja realizável, pois as divisões entre funções de transferências em (4) ou (5) podem resultar em sistemas não causais.

Tendo em vista que as funções de transferência do desacoplador são calculadas utilizando modelos FOPTD aproximados do sistema físico, sujeitos a ruídos e perturbações, a redução no acoplamento pode não ser efetiva. Uma forma de verificar e corrigir essa limitação consiste em avaliar o desacoplador e, se necessário, recalcular seus parâmetros. O procedimento adotado na avaliação e reprojeto é detalhado na seção 4 .

\subsection{Projeto de Controle PI Descentralizado}

No controle descentralizado, para a $i$-ésima malha SISO selecionada durante o emparelhamento das entradas e saídas, é sintonizado um controlador $C_{i}(s)$. A função de transferência do controlador PI na forma padrão é expressa por:

$$
C(s)=K_{p}\left(1+\frac{1}{T_{i} s}\right),
$$

onde $K_{p}$ é o ganho proporcional e $T_{i}$ a constante de tempo integral.

Nos testes realizados em malha fechada para observação do desempenho do desacoplador, foi utilizada a técnica de sintonia C-H-R para o problema servo, proposta por Chien et al. (1952), pela qual busca-se obter a reposta mais rápida possível sem sobressinal. O cálculo dos parâmetros para controladores PI são listados na Tabela 1.

Tabela 1. Sintonia dos controladores segundo o método C-H-R.

\begin{tabular}{ccc}
\hline Controlador & $K_{p}$ & $T_{i}$ \\
\hline PI & $\frac{0,35 T}{K L}$ & $1,16 T$
\end{tabular}

Onde $K, T$ e $L$ são os parâmetros do modelo FOPTD, expresso em (1), da malha em que será sintonizado o controlador.

Como no módulo de temperatura existe um ruído de medição significativo, a utilização de um controlador com efeito derivativo causaria sua amplificação. Por essa razão optou-se pelo controlador PI.

\section{AVALIAÇÃO E REPROJETO DO DESACOPLADOR}

Devido às incertezas contidas no modelo estimado do processo, o desempenho do desacoplador pode ser comprometido a depender das frequências dos sinais de entrada aplicados. Acioli Júnior (2012) e Aguiar (2018) estudaram metodologias para avaliação e reprojeto do desacoplador nas frequências $\omega=0$ e $\omega_{90}$ (frequência correspondente a fase de $90^{\circ}$ ). A principal diferença entre esssas metodologias é que na primeira é utilizada apenas a informação de $\omega_{90}$ e na segunda a frequência $\omega=0$ também é considerada.

As metodologias apresentadas são aplicáveis na avaliação e reprojeto do desacoplador invertido obtido para o módulo didático multivariável. Inicialmente, são avaliados os índices de erro na frequência do desacoplador projetado no item 3.3. Em seguida, é realizado o reprojeto a fim de minimizar o erro a partir do ajuste dos parâmetros do desacoplador. Neste artigo, será adotada a metodologia que utiliza os dois pontos de frequência (Aguiar et al., 2019). 


\subsection{Experimento para Avaliação do Desacoplador}

O método de avaliação utilizando os dois pontos de frequência supracitados para processos TITO é realizado a partir da aplicação de uma excitação na $j$-ésima malha do sistema desacoplado, observando a saída oposta, ou seja, da $i$-ésima malha $(i \neq j)$.

Para a avaliação nas frequências $\omega=0$ e $\omega_{90}$, o sinal de excitação consiste em uma onda quadrada de frequência $\omega_{90}^{i}$ de $G_{j j}$ seguido de um pulso com duração mínima de três períodos $2 \pi / \omega_{90}^{i}$ (Aguiar, 2018). Assim, é possível calcular o índice de erro nas frequências desejadas.

O índice de erro do desacoplamento na frequência $\omega^{i}$ para a $i$-ésima malha de um sistema TITO $\left(H_{i}(j \omega)\right)$ é dado por:

$$
H_{i}\left(j \omega^{i}\right)=\frac{Y_{i}\left(j \omega^{i}\right)}{U_{j}\left(j \omega^{i}\right)}=\frac{G_{i j}\left(j \omega^{i}\right)+G_{i i}\left(j \omega^{i}\right) D_{i j}\left(j \omega^{i}\right)}{1-D_{i j}\left(j \omega^{i}\right) D_{j i}\left(j \omega^{i}\right)},
$$

onde $i, j=1,2$ e $i \neq j$.

\subsection{Reprojeto do Desacoplador}

No caso em que valor do índice de erro de desacoplamento não é próximo de zero, o reprojeto é realizado para minimizá-lo ao determinar novos parâmetros de ganho ou constantes de tempo do desacoplador.

Sejam $D_{i j}^{0}(j \omega)$ e $D_{i j}^{1}(j \omega)$ as funções de transferência dos desacopladores inicial e reprojetado, respectivamente. A condição $H_{i}\left(j \omega^{i}\right) \approx 0$ é obtida com a solução de:

$$
D_{i j}^{1}\left(j \omega^{i}\right)-D_{i j}^{0}\left(j \omega^{i}\right)=-\frac{\Upsilon_{i}\left(j \omega^{i}\right)}{G_{i i}\left(j \omega^{i}\right)}
$$

onde

$$
\Upsilon_{i}\left(j \omega^{i}\right)=H_{i}\left(j \omega^{i}\right)\left(1-D_{i j}^{0}\left(j \omega^{i}\right) D_{j i}^{0}\left(j \omega^{i}\right)\right) .
$$

O cálculo dos novos parâmetros do desacoplador utilizando dois pontos de frequência é feito em duas etapas. A primeira consiste em modificar o ganho de forma a reduzir o índice de erro de desacoplamento na frequência $\omega=0$. O novo ganho $\left(K_{i}^{1}\right)$ é:

$$
K_{i}^{1}=\operatorname{Re}\left\{-\frac{\Upsilon_{i}(0)}{G_{i i}(0)}\right\}+K_{i}^{0}
$$

onde o operador $\operatorname{Re}\{\}$ retorna a parte real do argumento.

$\mathrm{Na}$ segunda etapa, uma das constantes de tempo do desacoplador é alterada para reduzir o índice de erro de desacoplamento na frequência $\omega_{90}$. Dessa forma, é possível escolher entre duas possibilidades de reprojeto. Na etapa 2.1, determina-se um novo valor da constante do denominador $\left(T_{i j}^{1}\right)$, expressa por:

$$
T_{i j}^{1}=\frac{1}{\omega_{90}^{i}} \operatorname{Im}\left\{\frac{K_{i}^{1}-\Gamma_{i}\left(j \omega_{90}^{i}\right)}{\Gamma_{i}\left(j \omega_{90}^{i}\right)}\right\}
$$

onde o operador $\operatorname{Im}\{\}$ retorna a parte imaginária do argumento e

$$
\Gamma_{i}\left(j \omega_{90}^{i}\right)=\frac{-\Upsilon_{i}\left(j \omega_{90}^{i}\right) / G_{i i}\left(j \omega_{90}^{i}\right)+D_{i j}^{0}\left(j \omega_{90}^{i}\right)}{\left(j \omega_{90}^{i} T_{i i}^{0}+1\right) e^{-j \omega_{90}^{i} \theta_{i}}} .
$$

Como o valor calculado de $T_{i j}^{1}$ pode ser negativo, o que resultaria em um polo instável, pode-se de maneira alternativa modificar a constante de tempo do numerador $\left(T_{i i}^{1}\right)$ (etapa 2.2). O valor de $T_{i i}^{1}$ é dado por:

$$
T_{i i}^{1}=\frac{1}{K_{i}^{1} \omega_{90}^{i}} \operatorname{Im}\left\{\Psi_{i}\left(j \omega_{90}^{i}\right)\right\}
$$

onde

$$
\Psi_{i}\left(j \omega_{90}^{i}\right)=\left[-\frac{\Upsilon_{i}\left(j \omega_{90}^{i}\right)}{G_{i i}\left(j \omega_{90}^{i}\right)}+D_{i j}^{0}\left(j \omega_{90}^{i}\right)\right]\left(j \omega_{90}^{i} T_{i j}^{0}+1\right) e^{j \omega_{90}^{i} \theta_{i}}
$$

\section{RESULTADOS EXPERIMENTAIS}

Nesta seção, são apresentados os resultados experimentais obtidos na aplicação da teoria de controle por desacoplamento invertido ao módulo didático de temperatura.

\subsection{Modelo Identificado do Processo}

O módulo de temperatura possui dinâmicas de aquecimento e resfriamento diferentes. Dessa forma, foram aplicados dois degraus em cada entrada, com amplitudes positiva e negativa, respectivamente. A partir desse experimento foram identificados modelos FOPTD distintos para subida e descida. O modelo utilizado para aplicação das estratégias de controle é constituído pelos valores médios dos parâmetros obtidos nos modelos de aquecimento e resfriamento.

Inicialmente, foi aplicado um valor de $40 \%$ a cada entrada individualmente para levar o sistema ao ponto de operação. Em seguida, foi realizado o teste do degrau utilizando amplitudes de 10\%. Na Fig. 3, são apresentados os gráficos das respostas dos sistema abservadas com a aplicação do experimento do degrau à cada entrada individualmente.

Nesse experimento, a matriz de transferência obtida foi:

$$
G(s)=\left[\begin{array}{ll}
\frac{39,75}{166,92 s+1} e^{-19,65 s} & \frac{20,12}{147,72 s+1} e^{-54,68 s} \\
\frac{28,70}{186,54 s+1} e^{-33,14 s} & \frac{30,98}{150,64 s+1} e^{-19,42 s}
\end{array}\right]
$$

\subsection{Emparelhamento de entradas e saídas}

A RGA calculada por meio de (2), utilizando o modelo expresso em (16) foi:

$$
\Lambda(G)=\left[\begin{array}{cc}
1,8829 & -0,8829 \\
-0,8829 & 1,8829
\end{array}\right]
$$

Visto que os termos da diagonal secundária são negativos, o pareamento nesse caso foi definido como $u_{1}-y_{1}$ para a malha 1 e $u_{2}-y_{2}$ para a malha 2 . 


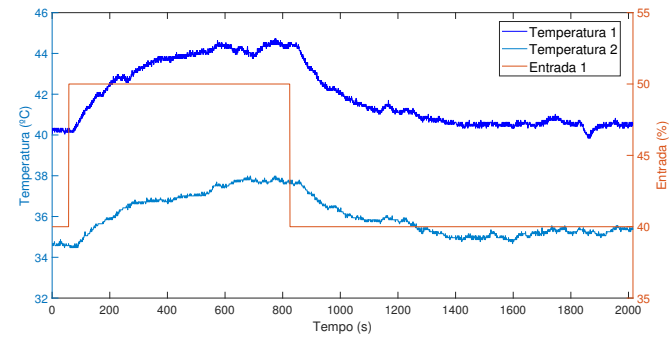

(a) Entrada 1

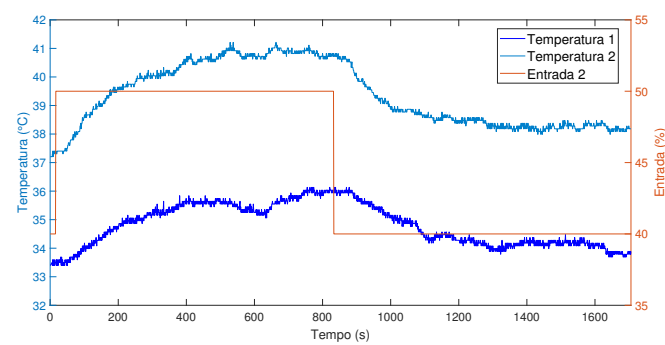

(b) Entrada 2

Figura 3. Experimento do degrau aplicado às entradas do sistema.

\subsection{Projeto do Desacoplador}

As funções de transferência do desacoplador, calculadas com base em (6), são expressas por:

$$
\begin{aligned}
& D_{12}(s)=-0,51\left(\frac{166,92 s+1}{147,72 s+1}\right) e^{-35,03 s}, \\
& D_{21}(s)=-0,93\left(\frac{150,64 s+1}{186,54 s+1}\right) e^{-13,72 s} .
\end{aligned}
$$

\subsection{Reprojeto do Desacoplador}

Tendo em vista que o módulo didático de temperatura é muito sensível a ruídos e perturbações externas, os parâmetros do modelo identificado contém incertezas. Como o desacoplador foi calculado a partir do modelo, é esperado que o desacoplamento não seja efetivo.

Dessa forma, foram aplicados os procedimentos de avaliação e reprojeto descritos na seção 4. Os parâmetros recalculados de ganho e constantes de tempo dos desacopladores $D_{12}$ e $D_{21}$ são mostrados na Tabela 2 .

Para comparar o desempenho dos desacopladores reprojetados, foi realizado o procedimento de avaliação descrito na seção 4.1 para cada modelo obtido no reprojeto. $\mathrm{Na}$ Tabela 3, são apresentadas as magnitudes dos índices de erro do desacoplamento nos dois pontos de frequência.

Tabela 3. Índices de erro nas frequências $\omega=0$ e $\omega_{90}$ do desacoplador invertido.

\begin{tabular}{lcccc}
\hline & $\left|H_{1}(0)\right|$ & $\left|H_{2}(0)\right|$ & $\left|H_{1}\left(j \omega_{90}^{1}\right)\right|$ & $\left|H_{2}\left(j \omega_{90}^{2}\right)\right|$ \\
\hline Inicial & 5,99 & 11,39 & 7,13 & 2,12 \\
Etapa 1 & 1,75 & 10,89 & 6,53 & 1,75 \\
Etapa 2.1 & 1,54 & 8,91 & 0,99 & 1,60 \\
Etapa 2.2 & 2,09 & 9,48 & 3,32 & 1,95 \\
\hline
\end{tabular}

Tabela 2. Parâmetros modificados dos desacopladores $D_{12}$ e $D_{21}$.

\begin{tabular}{lccc}
\hline$D_{12}$ & $K_{1}$ & $T_{11}$ & $T_{12}$ \\
\hline Inicial & $-0,51$ & 166,92 & 147,72 \\
Etapa 1 & $-0,66$ & 166,92 & 147,72 \\
Etapa 2.1 & $-0,66$ & 166,92 & 243,10 \\
Etapa 2.2 & $-0,66$ & 87,11 & 147,72 \\
\hline$D_{21}$ & $K_{2}$ & $T_{22}$ & $T_{21}$ \\
\hline Inicial & $-0,93$ & 150,64 & 186,54 \\
Etapa 1 & $-0,50$ & 150,64 & 186,54 \\
Etapa 2.1 & $-0,50$ & 150,64 & 123,70 \\
Etapa 2.2 & $-0,50$ & 187,60 & 186,54
\end{tabular}

Observou-se que os índices de erro na frequência foram reduzidos com o reprojeto do desacoplador. O melhor caso obtido foi com a etapa 2.1, onde foram modificados o ganho e a constante de tempo do denominador.

\subsection{Projeto dos Controladores PI}

As funções de transferência dos controladores PI $C_{i}(s)$, $i=1,2$ das malhas 1 e 2 , calculadas utilizando o modelo expresso em (7) com a aplicação do método C-H-R, são dadas por:

$$
\begin{aligned}
& C_{1}(s)=0,075\left(1+\frac{1}{183,63 s}\right) \\
& C_{2}(s)=0,088\left(1+\frac{1}{174,74 s}\right) .
\end{aligned}
$$

\subsection{Testes em Malha Fechada}

Com o objetivo de observar experimentalmente o comportamento do sistema em malha fechada com a inserção dos desacopladores projetados, foram realizados testes em malha fechada.

Inicialmente, foram aplicadas referências de $40^{\circ} \mathrm{C}$ às malhas 1 e 2 simultâneamente. Em seguida, variou-se a referência em $\pm 3^{\circ} C$ para cada malha separadamente. A resposta do sistema em malha fechada sem o desacoplador e com os desacopladores inicial e reprojetado (etapa 2.1), para cada malha, é apresentada nas Figs. 4 e 5.

Foi utilizado o erro médio ao quadrado como critério de comparação do desempenho dos desacopladores. Os resultados obtidos são apresentados na Tabela 4 .

Tabela 4. Erro médio ao quadrado calculado para o experimento em malha fechada.

\begin{tabular}{cccc}
\hline & Sem Desacoplador & Inicial & Etapa 2.1 \\
\hline Malha 1 & 0,253 & 0,040 & 0,077 \\
Malha 2 & 0,274 & 0,094 & 0,046 \\
\hline
\end{tabular}

Observou-se que a presença do desacoplador reduziu significativamente as variações na temperatura de cada malha decorrentes da mudança de referência da malha oposta. O desempenho dos desacopladores inicial e o reprojeto da etapa 2.1 foram semelhantes, sendo o primeiro mais eficaz na malha 1 e o segundo na malha 2 . 


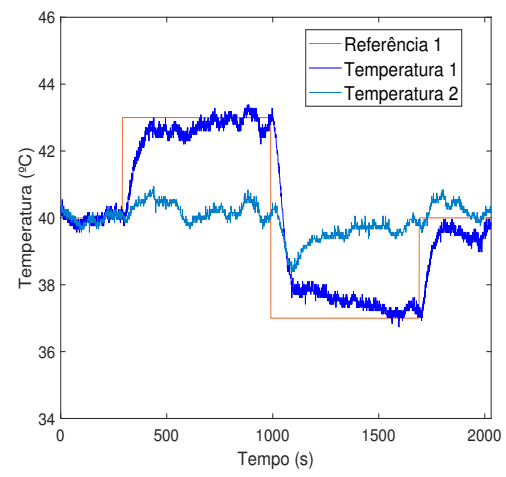

(a) Sem desacoplador.

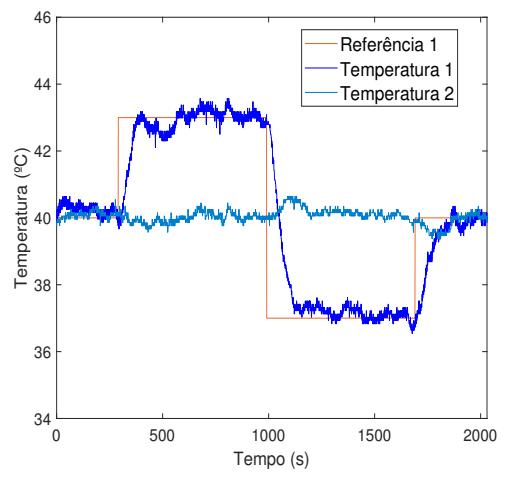

(b) Desacoplador inicial.

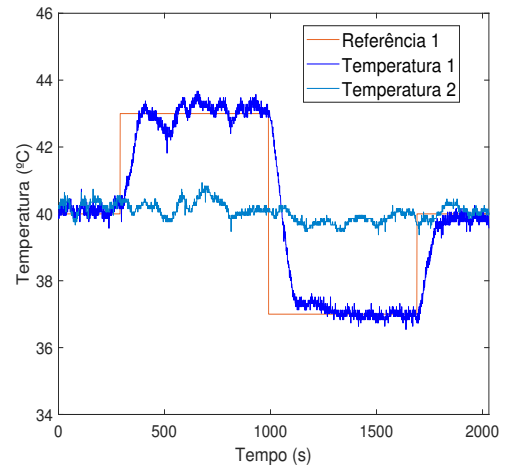

(c) Desacoplador reprojetado na etapa 2.1.

Figura 4. Desempenho em malha fechada do desacopladores inicial e reprojetado quando a referência da malha 1 é modificada.

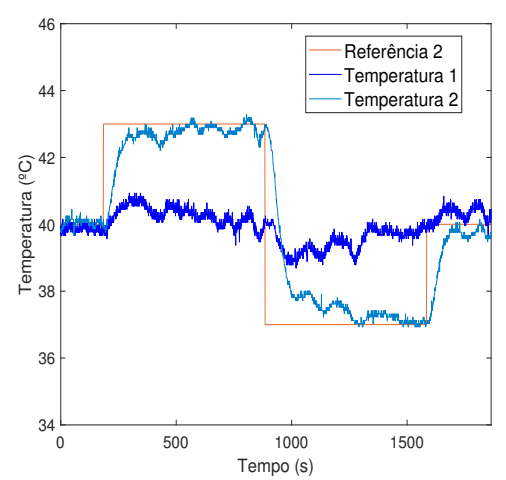

(a) Sem desacoplador.

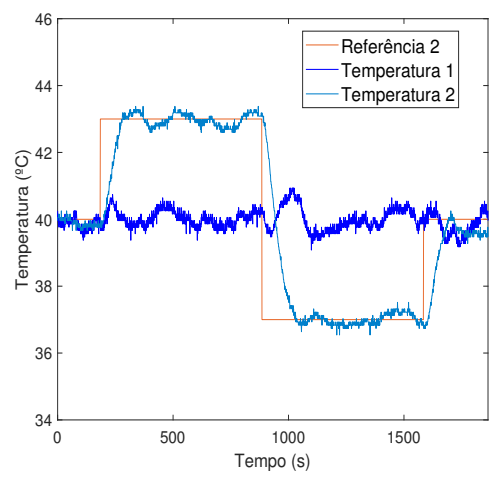

(b) Desacoplador inicial.

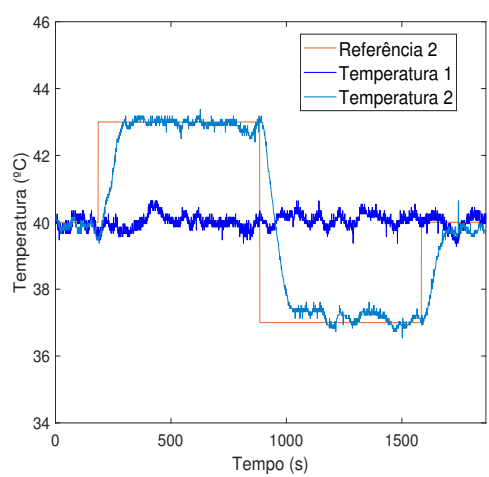

(c) Desacoplador reprojetado na etapa 2.1.

Figura 5. Desempenho em malha fechada dos desacopladores inicial e reprojetado quando a referência da malha 2 é modificada.

\section{CONCLUSÕES}

Neste trabalho, foi apresentada uma metodologia para realização de experimento. Esta permite aos alunos da graduação executarem o projeto de controle PI descentralizado por desacoplamento em um sistema multivariável. As etapas sugeridas consistem na identificação, seleção das malhas SISO, projeto, avaliação e reprojeto do desacoplador, bem como o projeto do controlador. As figuras e tabelas apresentadas na seção de resultados permitiram observar a eficácia do método estudado e a viabilidade de sua aplicação a um módulo didático, como o de temperatura utilizado.

\section{REFERÊNCIAS}

Acioli Júnior, G. (2012). Avaliação e Reprojeto para Controle PI de Sistemas Multivariáveis utilizando Estruturas de Realimentação com Relé. Ph.D. thesis, Universidade Federal de Campina Grande.

Acioli Júnior, G. and Barros, P.R. (2011). Evaluation and redesign of decouplers for tito processes using relay experiment. In IEEE International Conference on Control Applications (CCA), 1145-1150.

Aguiar, A.P.V.A. (2018). Projeto de Controle PID Descentralizado com Desacoplamento. Master's thesis, Universidade Federal de Campina Grande.
Aguiar, A.P.V.A., Acioli Júnior, G., and Barros, P.R. (2019). Frequency domain evaluation and redesign of inverted decoupler. In 12th IFAC Symposium on Dynamics and Control of Process Systems.

Bristol, E. (1966). On a new measure of interaction for multivariable process control. IEEE transactions on automatic control, 11(1), 133-134.

Chien, K.L., Hrones, J.A., and Reswick, J.B. (1952). On the automatic control of generalized passive systems. Transactions of ASME, 74, 175-185.

Dong, J., Sun, L., Li, D., Lee, K.Y., and Wu, Z. (2015). Inverted decoupling based active disturbance rejection control foi multivariable systems. In IEEE 54th Annual Conference on Decision and Control (CDC), 7353-7358.

Lima, A.B.D., Barros, P.R., and Acioli Júnior, G. (2018). Módulo didático para ensino de teoria de controle. In Congresso Brasileiro de Automática (CBA).

Luyben, W.L. (1970). Distillation decoupling. AIChE Journal, 16, 198-203.

Shinskey, F.G. (1996). Process control systems: application, design and tuning. McGraw-Hill.

Wang, Q.G. (2002). Decoupling Control. Springer, 1 edition. 